\title{
EFFICIENT CALCULATION OF THE RESPONSE OF A STRUCTURAL ACOUSTIC SYSTEM UNDER AERODYNAMIC EXCITATIONS
}

\author{
D. Chronopoulos ${ }^{1,2}$, M. Ichchou ${ }^{2}$, and B. Troclet $^{1}$ \\ ${ }^{1}$ EADS Astrium ST \\ 66 Route de Verneuil, BP3002, 78133 Les Mureaux Cedex, France \\ e-mail: chronopoulos.dimitri@gmail.com \\ ${ }^{2}$ Ecole Centrale de Lyon \\ 36 Avenue Guy de Collongue, 69134 Ecully Cedex, France
}

Keywords: Fluid-structure interaction, Composite structures, Krylov-subspace methods, Random excitations

\begin{abstract}
The problem of the dynamic response of a structural-acoustic system in the midfrequency range is hereby considered. The system is initially modelled using finite elements, and is subsequently reduced using the Second Order Arnoldi Reduction method (SOAR) resulting in radical reduction of calculation times. The fully coupled system is modelled using a Statistical Energy Analysis like (SEA-like) approach, and the energetic characteristics for each subsystem are computed and compared to the direct FEM solution. The error with respect to the full FE solution is presented and the limits of the reliability of the reduction are explored. The loading applied to the model comprises typical random aeroacoustic excitations, such as a diffused sound field and a Turbulent Boundary Layer (TBL) excitation.
\end{abstract}




\section{INTRODUCTION}

Structural acoustic interactions in a broadband frequency range are a regular problem in modern aerospace and automotive industry. The level of noise induced by structural vibration in a cavity has to be predicted, in order to optimize the acoustic transparency of a system, such as a payload cavity of a launch vehicle, or a passenger cavity for a car. The structural loading usually includes random, distributed excitations, such as diffused acoustic fields and aerodynamic excitations. The structures are often made of composite non-isotropic materials, complicating the modelling of the structural response. Moreover, the size of the cavities is often too large, which makes deterministic techniques such as Finite Elements (FE) excessively expensive in terms of computational time at higher frequencies [1]. Statistical methods such as Statistical Energy Analysis (SEA) are often considered more suitable for the medium frequency range but have limited accuracy, particularly when the modal overlap of the system is low [2]. It is therefore a vital task, the development of a robust approach for the calculation of the response of large systems, in that range where deterministic methods are too costly and statistical methods too inaccurate.

Numerous authors have investigated the fluid-structure interaction in the broadband frequency domain. In the low-frequency range, the system is typically modelled using FE or Boundary Elements (BE) as in [3]. A Component Mode Synthesis (CMS) was used in [4] in order to predict the low-frequency one-dimension fluid-structure interaction. The authors in [5] gave an energetic description of a panel-cavity coupled system in the mid-frequency range, by considering the modes of each uncoupled subsystem. The work was extended by Rochambeau et al. using an SEA-like approach in [6], in order to give an accurate description of the fluidstructure interaction problem in the mid-frequency range. The method was initially introduced and studied by the authors in $[2,7,8,9]$. The impact of the strength of coupling between the structure and the fluid on the accuracy of the prediction is investigated in [6]. Strong coupling means that taking into account for the uncoupled modes of the subsystems will lead to inaccurate results especially for the low frequency range. Furthermore the assumption of analytical expressions of the modal data of the subsystems used in [6] restricts the applicability of the method to rectangular thin isotropic structures and rectangular cavities.

In order to reduce the computational effort required to resolve a structural-acoustic system, several reduction methods have been introduced. Modal reduction techniques have been successfully applied in $[6,10,11]$, but they usually require analytical expressions of the modal data of the subsystems which are not always available (e.g for composite structures of arbitrary geometry). Recently, the moment matching approaches for the reduction of large scale second order systems have received much attention $[12,13,14,15]$. The method consists in finding a reduced Krylov subspace to project the initial system and was successfully applied to a fully coupled structural-acoustic system in the low-frequency range in [15]. Bai and Su in [16] introduced the second-order Krylov subspace and the Second Order Arnoldi procedure (SOAR) in order for the reduced system to maintain the second order form of the initial one.

The modelling of pragmatic aerodynamic loads have been a long-term challenge. The author in [17] presented one of the first models for a Turbulent Boundary Layer (TBL) excitation. Other models derived from Corcos expression were then suggested by Chase and Efimstov in order to improve the predictions for the low-frequency range $[18,19]$. In order for such loads to be integrated in an SEA type method, $\delta$-correlated equivalent expressions for the excitations are required. Maidanik was the first to give such an expression, with the spatial-extent approach in [20]. Recently, equivalent approaches were suggested based on a wavenumber expression of 


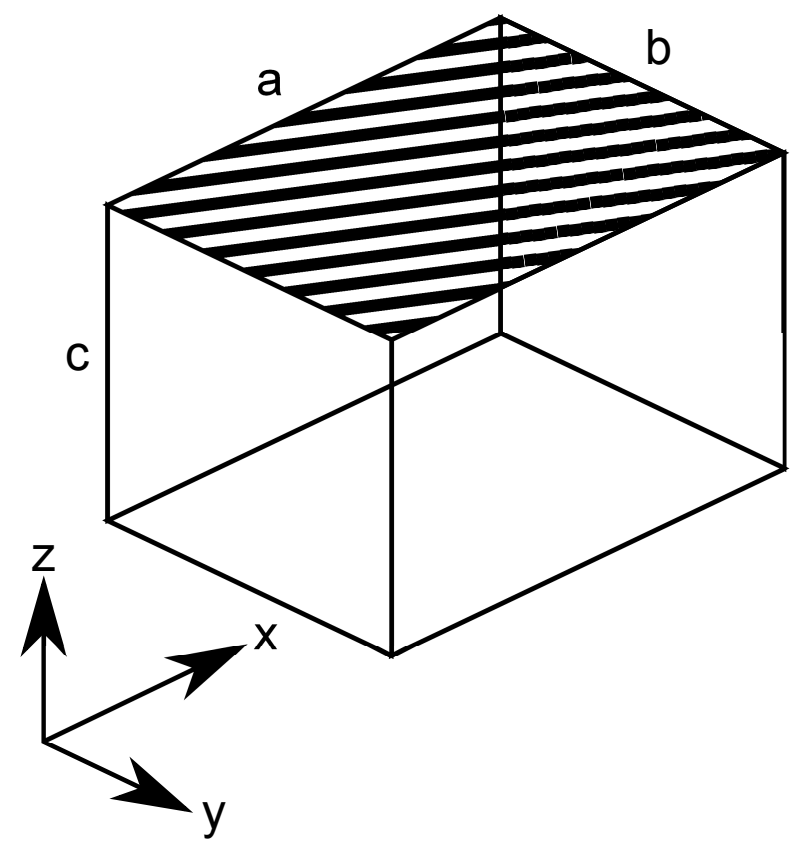

Figure 1: View of the modelled configuration

the excitation. The author in [21] modelled a TBL excitation using an asymptotic approach for the wavenumber transform of the spatial coherence function. In [22], the authors applied the equivalent $\delta$-correlated excitation method on a rectangular panel and proposed an equivalent model for a TBL excitation.

In this work the use of a second order moment matching method in order to reduce a fully coupled structural-acoustic system, subject to realistic aerodynamic distributed excitations in a broadband frequency range is presented. An expression for the TBL equivalent coherence function when applied on an orthotropic plate is also given. The approach proves to be generic and robust. It drastically reduces the computation times. Moreover the advantages of an FE modelling are maintained. This implies that the system can be of arbitrary geometry and the structure can be made of composite nature. The coupling between the subsystems can also be of arbitrary nature.

\section{Modelling a structural-acoustic system with a hybrid FE/SEA method}

A vibroacoustic system as the one presented in fig. 1 is to be modelled. In the mid-frequency domain FE modelling becomes greatly costly in terms of computational times. On the other hand, the conditions for forming a proper SEA matrix are not always satisfied, mainly because of the fact that the weak coupling condition between the subsystems is not satisfied and because of the indirect coupling between substructures that cannot be neglected. Moreover, in the midfrequency range the intrinsic damping of the substructures has a great role on energetic transfer between them. Consequently, damping cannot be separated from the Coupling Loss Factors (CLF), as it is in SEA equations. 
The Energy Influence Coefficient (EIC) method was presented in [23]. As in SEA, the system is discretized in distinct $n$ interconnected subsystems. Assuming that the loads applied to different subsystems are uncorrelated, and that the loads applied to each subsystem are $\delta$ correlated, the column matrix of subsystems kinetic energies can be written as the product of the EIC matrix with the matrix of Power Spectral Densities (PSD) of the loads applied to the subsystems:

$$
\left\{\begin{array}{c}
E_{1} \\
E_{2} \\
\ldots \\
E_{n}
\end{array}\right\}=\left[\begin{array}{cccc}
A_{11} & A_{12} & \ldots & A_{1 n} \\
A_{21} & A_{22} & \ldots & A_{2 n} \\
\ldots & \ldots & \ldots & \ldots \\
A_{n 1} & A_{n 2} & \ldots & A_{n n}
\end{array}\right]\left\{\begin{array}{c}
P_{1} \\
P_{2} \\
\ldots \\
P_{n}
\end{array}\right\}
$$

The SEA-like method used in [7, 8, 9], is very similar to the EIC method, with the sole difference being that the kinetic energy of the subsystems is replaced by their total energy. By exciting the subsystems one by one, the above relation can be written as:

$$
\left[\begin{array}{cccc}
\left\langle E_{11}\right\rangle & \left\langle E_{12}\right\rangle & \ldots & \left\langle E_{1 n}\right\rangle \\
\left\langle E_{21}\right\rangle & \left\langle E_{22}\right\rangle & \ldots & \left\langle E_{2 n}\right\rangle \\
\ldots & \ldots & \ldots & \ldots \\
\left\langle E_{n 1}\right\rangle & \left\langle E_{n 2}\right\rangle & \ldots & \left\langle E_{n n}\right\rangle
\end{array}\right]=\left[\begin{array}{cccc}
A_{11} & A_{12} & \ldots & A_{1 n} \\
A_{21} & A_{22} & \ldots & A_{2 n} \\
\ldots & \ldots & \ldots & \ldots \\
A_{n 1} & A_{n 2} & \ldots & A_{n n}
\end{array}\right] \mathbf{I}
$$

where

$$
\left\langle E_{i j}\right\rangle=\frac{E_{i j}}{P_{j}}
$$

with $E_{i j}$ the total energy of subsystem $i$ when subsystem $j$ is excited and $P_{j}$ the PSD injected in subsystem $j$. Finally, the EIC matrix can be written as:

$$
\left[\begin{array}{cccc}
A_{11} & A_{12} & \ldots & A_{1 n} \\
A_{21} & A_{22} & \ldots & A_{2 n} \\
\ldots & \ldots & \ldots & \ldots \\
A_{n 1} & A_{n 2} & \ldots & A_{n n}
\end{array}\right]=\left[\begin{array}{cccc}
\left\langle E_{11}\right\rangle & \left\langle E_{12}\right\rangle & \ldots & \left\langle E_{1 n}\right\rangle \\
\left\langle E_{21}\right\rangle & \left\langle E_{22}\right\rangle & \ldots & \left\langle E_{2 n}\right\rangle \\
\ldots & \ldots & \ldots & \ldots \\
\left\langle E_{n 1}\right\rangle & \left\langle E_{n 2}\right\rangle & \ldots & \left\langle E_{n n}\right\rangle
\end{array}\right]
$$

The injected power as well as the subsystem energies can be computed, by an FE modelling. The spatially averaged calculated values will then be used to estimate the energy of each subsystem and the energy exchange between them. Classic FE models as in [24] will be used for the structural acoustic coupling. The total energy of an acoustic subsystem comprising $N$ nodes can be written as:

$$
E_{a c}(\omega)=\frac{\left\langle p_{i}^{2}\right\rangle V}{\rho_{0} c_{0}^{2}}
$$

with $\left\langle p_{i}^{2}\right\rangle$ the mean-square sound pressure of subsystem $i, V$ the volume of the cavity, $\rho_{0}$ the density of the acoustic medium and $c_{0}$ the celerity of the medium. The total energy of a structural subsystem can be written as:

$$
E_{\text {str }}(\omega)=\rho_{s} S\left\langle\tilde{v}_{i}^{2}\right\rangle
$$

assuming that the potential and kinetic energies in both types of subsystems are equal. In eq.(6), $\left\langle\tilde{v}_{i}^{2}\right\rangle$ is the mean-square velocity of subsystem $i, \rho_{s}$ is the surface density of the structure and $S$ is the total area of the structural component. 


\section{Random distributed excitations}

As mentioned in sec.2, in order to calculate the EIC of a system using the SEA-like method, a $\delta$-correlated 'Rain-On-the-Roof' (ROR) excitation has to be applied on the subsystems. Moreover, the excitations applied to different subsystems have to be uncorrelated. Unluckily however, ROR does not represent real world excitations.

The most commonly encountered random aeroacoustic excitations in the aerospace and automotive fields are either diffused acoustic fields, or TBL excited vibration during high speed cruising. In [6] it is shown that the following relationships between an aeroacoustic excitation and a ROR one are valid:

$$
\begin{aligned}
& E_{s, s}^{a e r}(\omega) \approx \frac{C_{e q}(\omega)}{\rho_{s}} E_{s, s}^{r o r}(\omega) \\
& E_{a, s}^{a e r}(\omega) \approx \frac{C_{e q}(\omega)}{\rho_{s}} E_{a, s}^{r o r}(\omega) \\
& P_{s}^{a e r}(\omega) \approx \frac{C_{e q}(\omega)}{\rho_{s}} P_{s}^{r o r}(\omega)
\end{aligned}
$$

in which $s$ stands for the structure and $a$ for the acoustic medium. Therefore:

$$
\begin{aligned}
& A_{s, s}^{a e r} \approx A_{s, s}^{r o r} \\
& A_{a, s}^{a e r} \approx A_{a, s}^{r o r}
\end{aligned}
$$

Where $C_{e q}(\omega)$ is the equivalent coherence function of the aeroacoustic excitation. The great advantage of this formulation, is based on the fact that by calculating the energy exchange properties of the system under a ROR excitation, an aeroacoustic excitation can be simulated through its equivalent coherence function. Therefore only one simulation is needed for the prediction of the response of a system, under several simultaneously applied aerodynamic excitations.

\subsection{Equivalent function for a diffused sound field}

An incident diffused sound field comprises an infinite number of uncorrelated plane waves with incidence angles uniformly distributed over a half space. The equivalence correlation function of a diffused sound field can be written as in [6]:

$$
C_{\text {diff }}(\omega)=\left(\frac{\pi}{k_{0}}\right)^{2} \sigma_{\text {rad }}(\omega)
$$

Where $k_{0}$ is the acoustic wavenumber given by $k_{0}=\omega / c_{0}$. The radiation efficiency $\sigma_{\text {rad }}(\omega)$ for an orthotropic panel having finite dimensions can be calculated using expressions such as the set of asymptotic formulas given in [25].

\subsection{Equivalent function for a TBL excitation}

Following the wavenumber space equivalence approach presented in [22], an equivalent ROR excitation can be calculated. The equivalent correlation function can be expressed in the wavenumber space as:

$$
C_{e q}\left(k_{x}, k_{y}, \omega\right)=\frac{C_{e q}(\omega)}{4 \pi^{2}}
$$

Comparing the wavenumber-frequency spectrum of the studied excitation with the one of the equivalent ROR excitation, $C_{e q}$ can be written in cartesian coordinates as: 


$$
\int_{k_{x 1}}^{k_{x 2}} \int_{k_{y 1}}^{k_{y 2}} C_{e q}\left(k_{x}, k_{y}, \omega\right) d k_{y} d k_{x}=\int_{k_{x 1}}^{k_{x 2}} \int_{k_{y 1}}^{k_{y 2}} C\left(k_{x}, k_{y}, \omega\right) d k_{y} d k_{x}
$$

Substituting eq.(10) in eq.(11) implies that for an orthotropic panel $C_{e q}$ can be written as:

$$
C_{e q}(\omega)=\frac{4 \pi^{2}}{\left(k_{x 2}-k_{x 1}\right)\left(k_{y 2}-k_{y 1}\right)} \int_{k_{x 1}}^{k_{x 2}} \int_{k_{y 1}}^{k_{y 2}} C\left(k_{x}, k_{y}, \omega\right) d k_{y} d k_{x}
$$

A classic model presented in [17] will be used hereby, according to which the coherence function for a TBL excitation propagating in the $x$ direction is given as:

$$
C_{T B L}(\zeta, \eta, \omega)=e^{-\alpha_{x} \frac{\omega|\zeta|}{U_{c}}} e^{-\alpha_{y} \frac{\omega|\eta|}{U_{c}}} e^{\frac{i \omega \zeta}{U_{c}}}
$$

where $\zeta=x-x^{\prime}$ and $\eta=y-y^{\prime}$. The above expression can be written in the wavenumber space (see [6]) as:

$$
C_{T B L}\left(k_{x}, k_{y}, \omega\right)=\frac{\alpha_{x} \alpha_{y} k_{c}^{2}}{\pi^{2}\left(\alpha_{x}^{2} k_{c}^{2}+\left(k_{x}-k_{c}\right)^{2}\right)\left(a_{y}^{2} k_{c}^{2}+k_{y}^{2}\right)}
$$

Eventually, substituting eq.(14) in eq.(12) the equivalent coherence function of the TBL excitation can be obtained:

$$
C_{e q}(\omega)=\frac{4 \pi^{2}}{\left(k_{x 2}-k_{x 1}\right)\left(k_{y 2}-k_{y 1}\right)} \int_{k_{x 1}}^{k_{x 2}} \int_{k_{y 1}}^{k_{y 2}} \frac{\alpha_{x} \alpha_{y} k_{c}^{2}}{\pi^{2}\left(\alpha_{x}^{2} k_{c}^{2}+\left(k_{x}-k_{c}\right)^{2}\right)\left(a_{y}^{2} k_{c}^{2}+k_{y}^{2}\right)} d k_{y} d k_{x}
$$

In the above equations $a_{x}, a_{y}$ are empirical coefficients depending on the nature of the TBL excitation, $U_{c}$ is the convection velocity and $k_{c}=\omega / U_{c}$ is the convection wavenumber.

\section{Reduction using a second order moment matching method}

Following classical FE formulation for structural-acoustic coupled subsystems [24, 26], the discretized system can be written as a system of second order ordinary differential equations as:

$$
\begin{gathered}
\mathbf{M}\left\{\begin{array}{c}
\ddot{\tilde{x}}(t) \\
\ddot{\tilde{p}}(t)
\end{array}\right\}+\mathbf{C}\left\{\begin{array}{c}
\dot{\tilde{x}}(t) \\
\dot{\tilde{p}}(t)
\end{array}\right\}+\mathbf{K}\left\{\begin{array}{c}
\tilde{x}(t) \\
\tilde{p}(t)
\end{array}\right\}=\mathbf{F}_{\mathbf{s a}} \tilde{u}(t) \\
\tilde{y}(t)=\ell^{t}\left\{\begin{array}{c}
\tilde{x}(t) \\
\tilde{p}(t)
\end{array}\right\}
\end{gathered}
$$

For a system with $N$ DoF, $\mathbf{M}, \mathbf{C}, \mathbf{K} \in \Re^{N \times N}$ are the non-symmetric mass, damping and stiffness matrices, $\tilde{x}(t)$ stands for the structural displacement vector, $\tilde{p}(t)$ denotes the nodal pressure vector in the acoustic fluid, $\mathbf{F}_{\mathbf{s a}}$ is the input matrix, $\tilde{u}(t)$ signifies the load vector of the system and $\ell^{t}$ is the transposed output matrix.

\subsection{The Second Order ARnoldi (SOAR) process}

The objective of a model order reduction process is the calculation of a subspace $\mathbf{S} \in \mathbb{C}^{N \times m}$ with $m$ the size of the reduced model, for which the solution of the original system is written as:

$$
\left\{\begin{array}{l}
\tilde{x} \\
\tilde{p}
\end{array}\right\}=\mathbf{S} \tilde{b}+\tilde{\epsilon}
$$


with $\tilde{b} \in \Re^{m}$ and $\tilde{\epsilon}$ the error of the approximation process. Both $m$ and $\tilde{\epsilon}$ have to be minimized, for the computational cost and the error of the reduced solution to be acceptable. Assuming harmonic excitation, the system of equations can be written after a Laplace transform as:

$$
\begin{gathered}
s^{2} \mathbf{M}\left\{\begin{array}{c}
\tilde{x} \\
\tilde{p}
\end{array}\right\}+s \mathbf{C}\left\{\begin{array}{c}
\tilde{x} \\
\tilde{p}
\end{array}\right\}+\mathbf{K}\left\{\begin{array}{l}
\tilde{x} \\
\tilde{p}
\end{array}\right\}=\tilde{F} \\
\tilde{y}=\ell^{t}\left\{\begin{array}{c}
\tilde{x} \\
\tilde{p}
\end{array}\right\}
\end{gathered}
$$

After some algebraic manipulation of the above system the variable vector is eliminated and the transfer function can be written as:

$$
\tilde{h}(s)=\ell^{t}\left(s^{2} \mathbf{M}+s \mathbf{C}+\mathbf{K}\right)^{-1} \tilde{F}
$$

With $s=i \omega, \omega>0$. Assuming $\mathbf{K}$ non-singular the system has a Taylor series expansion which around $s=0$ can be written as in [27]:

$$
\tilde{h}(s)=\tilde{m}_{0}+\tilde{m}_{1} s+\tilde{m}_{2} s^{2}+\ldots=\sum_{i=1}^{\infty} \tilde{m}_{i} s^{i}
$$

with $\tilde{m}_{i}$ the leading moments. The idea of a moment matching method is to find a reduced system of dimension $m$, whose transfer function matches as many leading moments of the original system as possible. The second order Krylov subspace was introduced for this reason in [28]. It is defined as the subspace, spanned by the vector sequence $\tilde{q}_{i}$, denoted as:

$$
\mathcal{K}_{m}\left(\mathbf{A}, \mathbf{B}, \tilde{q}_{0}\right)=\operatorname{span}\left(\tilde{q}_{0}, \tilde{q}_{1}, \ldots, \tilde{q}_{m-1}\right)
$$

with the vector sequence $\tilde{q}_{i}$ :

$$
\tilde{q}_{i}=\left\{\begin{array}{c}
\tilde{q}_{0} \\
\mathbf{A} \tilde{q}_{0} \\
\mathbf{A} \tilde{q}_{i-1}+\mathbf{B} \tilde{q}_{i-2} \quad \text { for } i \geq 2
\end{array}\right.
$$

Vector $\tilde{q_{0}}$ is called the starting vector, while vectors $\tilde{q}_{i}$ for $i>0$ are called basic vectors. It is shown, [29] that for $\mathbf{A}=-\mathbf{K}^{-1} \mathbf{C}, \mathbf{B}=-\mathbf{K}^{-1} \mathbf{M}$, and $\tilde{q_{0}}=\mathbf{K}^{-1} \tilde{F}$, the moments of the system can be written with respect to the above vector sequence as:

$$
\tilde{m}_{i}=\ell^{t} \tilde{q}_{i}
$$

Assuming an orthogonal basis $\mathbf{S}$ of $\mathcal{K}_{m}$ and projecting the initial system on this basis we get the reduced second order system:

$$
\begin{gathered}
s^{2} \mathbf{M}_{\mathbf{r}} \tilde{b}+s \mathbf{C}_{\mathbf{r}} \tilde{b}+\mathbf{K}_{\mathbf{r}} \tilde{b}=\tilde{F}_{r} \\
\tilde{y}_{r}=\ell_{r}^{t} \tilde{b}
\end{gathered}
$$

with

$$
\mathbf{M}_{\mathbf{r}}=\mathbf{S}^{t} \mathbf{M S}, \mathbf{C}_{\mathbf{r}}=\mathbf{S}^{t} \mathbf{C S}, \mathbf{K}_{\mathbf{r}}=\mathbf{S}^{t} \mathbf{K S}, \tilde{F}_{r}=\mathbf{S}^{t} \tilde{F}, \ell_{r}^{t}=\ell^{t} \mathbf{S}
$$

In [30] it is shown that if a matrix $\mathbf{S}$ is a basis of the second-order Krylov subspace 


$$
\mathcal{K}_{m}\left(-\mathbf{K}^{-1} \mathbf{C},-\mathbf{K}^{-1} \mathbf{M}, \mathbf{K}^{-1} \tilde{F}\right)
$$

and $\mathbf{K}_{\mathbf{r}}$ is non-singular, then the first $m$ leading moments of the original and the reducedorder models are matched. The iterative process followed for finding an orthogonal basis $\mathbf{S}$ of the second order Krylov matrix was presented in [16], and is called a Second Order ARnoldi reduction (SOAR). The main advantage of SOAR compared to the Arnoldi reduction is the preservation of the nature of a second order system. It is therefore expected to be at least as accurate as the first order Arnoldi process [31].

\subsection{Expansion about $s_{0} \neq 0$}

If the expansion series of the system's transfer function is to be calculated around a point $s_{0} \neq 0$, eq.(19) can be written as:

$$
\begin{array}{r}
\tilde{h}(s)=\ell^{t}\left(\left(s+s_{0}\right)^{2} \mathbf{M}+\left(s+s_{0}\right) \mathbf{C}+\mathbf{K}\right)^{-1} \tilde{F} \\
=\ell^{t}\left(s^{2} \mathbf{M}+s\left(\mathbf{C}+2 s_{0} \mathbf{M}\right)+\left(\mathbf{K}+s_{0} \mathbf{C}+s_{0}^{2} \mathbf{M}\right)\right)^{-1} \tilde{F}
\end{array}
$$

By comparing eq.(19) to eq.(27), it can be seen that we can calculate the transfer function of a system around $s_{0}$ by substituting $\mathbf{K}$ with $\mathbf{K}+s_{0} \mathbf{C}+s_{0}^{2} \mathbf{M}$ and $\mathbf{C}$ with $\mathbf{C}+2 s_{0} \mathbf{M}$ in eq.(19). The modified Krylov subspace can therefore be written as:

$\mathcal{K}_{m}\left(\left(\mathbf{K}+s_{0} \mathbf{C}+s_{0}^{2} \mathbf{M}\right)^{-1}\left(\mathbf{C}+2 s_{0} \mathbf{M}\right),\left(\mathbf{K}+s_{0} \mathbf{C}+s_{0}^{2} \mathbf{M}\right)^{-1} \mathbf{M},\left(\mathbf{K}+s_{0} \mathbf{C}+s_{0}^{2} \mathbf{M}\right)^{-1} \tilde{F}\right)(28)$

and the modified reduced system matrices will be calculated by projecting the initial system's matrices to an orthogonal basis of the modified Krylov subspace.

\section{Numerical examples}

In this section, numerical applications for the models presented in sec. 2 to sec. 4 are exhibited.

The configuration shown in fig. 1 is to be modelled. The dimensions $a, b$ and $c$ are $0.7 \mathrm{~m}, 0.6 \mathrm{~m}$ and $0.5 \mathrm{~m}$ respectively. The structure is a layered, highly orthotropic panel made of Material I (see Table 1), with a thickness of $0.5 \mathrm{~mm}$. The damping of the system is considered to be Rayleigh proportional.

The structural-acoustic system is modelled by coupling Mindlin-Reissner type two-dimensional finite elements for the panel, to three-dimensional acoustic elements for the cavity. The resulting FE model comprises 3186 structural DOF and 17832 acoustic pressure DoF. A ROR excitation is initially applied to the structural panel, using loads of equal magnitude and of random phase and position. In order to excite the entirety of the subsystem modes in each frequency band (necessary condition for a ROR excitation), the Influence Circle approach exhibited in [9] will be used to decide the number of applied loads. The error $\epsilon(\omega)$ between the FE direct solution and the estimation of the reduced model is computed as:

$$
\tilde{\epsilon}(\omega)=\frac{\left|\tilde{h}_{r}(\omega)-\tilde{h}(\omega)\right|}{|\tilde{h}(\omega)|}
$$


Table 1: Mechanical properties of materials

\begin{tabular}{ccc}
\hline \hline Material I & Material II & Material III \\
\hline$\rho=9740 \mathrm{~kg} / \mathrm{m}^{3}$ & $\rho=1410 \mathrm{~kg} / \mathrm{m}^{3}$ & $\rho=48 \mathrm{~kg} / \mathrm{m}^{3}$ \\
$E_{x}=2023.7 \mathrm{GPa}$ & $E_{c}=54 \mathrm{GPa}$ & $E_{c}=85 \mathrm{MPa}$ \\
$E_{y}=31375 \mathrm{GPa}$ & $E_{a}=54 \mathrm{GPa}$ & $E_{a}=85 \mathrm{MPa}$ \\
$v_{x y}=0.028$ & $v_{c a}=0.09$ & $v_{c a}=0.23$ \\
$v_{x z}=0$ & $v_{c r}=0.09$ & $v_{c r}=0.23$ \\
$v_{y z}=0.434$ & $v_{a r}=0.09$ & $v_{a r}=0.23$ \\
$G_{x y}=888.79 \mathrm{GPa}$ & $G_{c a}=8.5 \mathrm{GPa}$ & $G_{c a}=49 \mathrm{MPa}$ \\
$G_{y z}=888.79 \mathrm{GPa}$ & $G_{a r}=8.5 \mathrm{GPa}$ & $G_{a r}=44 \mathrm{MPa}$ \\
$G_{x z}=888.79 \mathrm{GPa}$ & $G_{c r}=8.5 \mathrm{GPa}$ & $G_{c r}=24 \mathrm{MPa}$ \\
\hline
\end{tabular}

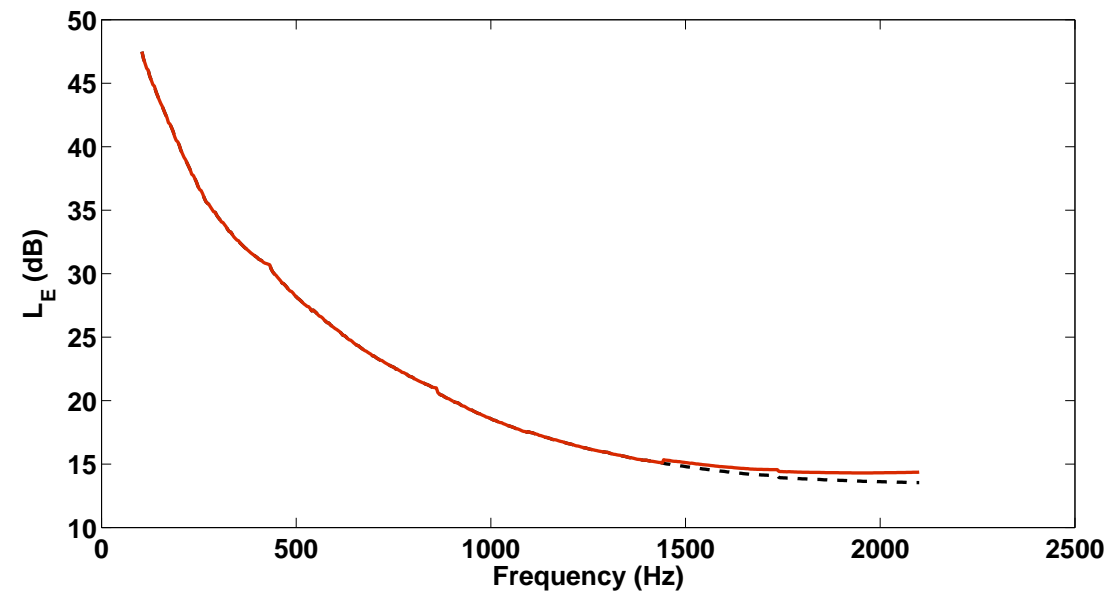

Figure 2: Steady-state energy for the structural subsystem under a reverberant field excitation: FE direct solution $(--)$, SOAR method (-)

\subsection{Diffused field excitation}

The results of an SEA-like analysis with the panel excited by a reverberant field on its dry side are presented hereby. As stated in sec.3, a new FE simulation is not needed for the response of the system to be predicted. The energy quantities of the subsystems under a diffused field excitation, are directly related to the ones for a ROR (of an equal Power Spectral Density (PSD)) through the equivalence function presented in eq.(9). The radiation efficiency of the panel is computed using the Leppington's set of asymptotic formulas, accounting for the finite dimensions, as well as the orthotropy of the panel. The resulting levels of energy $L_{E}$ for the subsystems are presented in fig.2,3.

The comparison between the FE and SOAR methods are in very good agreement for the entire frequency range. The maximum difference between the predictions occurs at the end of the analysis range for both subsystems and is equal to $0.9 \mathrm{~dB}$ for the panel's and $0.7 \mathrm{~dB}$ for the cavity's energy level.

\subsection{TBL excitation}

A TBL excitation with empirical values corresponding to $U_{c}=0.9$ Mach which is a typical convection velocity for an aerospace structure, will be modelled using its equivalent coherence function presented in eq.(15). A stream propagating in $x$, as well as in $y$ direction is hereby 


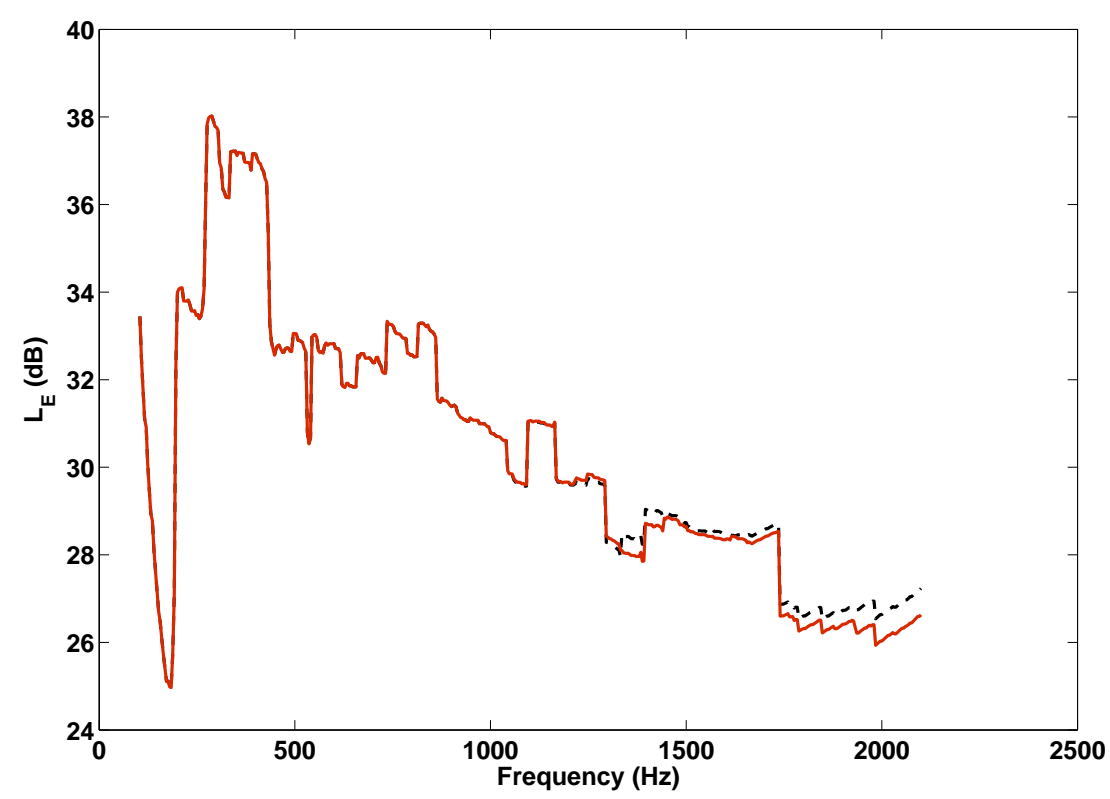

Figure 3: Steady-state energy for the acoustic subsystem under a reverberant field excitation: FE direct solution $(--)$, SOAR method (-)

modelled. The resulting acoustic energy inside the cavity subsystem is shown in fig.4.

It is shown that the FE results are in a very good agreement with the ones obtained after the SOAR reduction. The largest discrepancy for both results is $0.6 \mathrm{~dB}$ at $f=2100 \mathrm{~Hz}$. The different resulting energies for the two propagation directions are due to the orthotropic characteristics of the panel. The higher induced energy for the stream propagating towards $y$ direction implies that the convective wavenumber is closer to the panel's structural wavenumber in this direction, resulting in better coupling and more efficient energy transmission.

\subsection{Predicting the sound level within a spacecraft's payload cavity}

In this section the sound level within an empty payload cavity of the Ariane 5 spacecraft is to be predicted under a ROR and a TBL excitation. The configuration to be vibroacoustically computed is the SYLDA structure; its name standing for the french acronym of SYstéme de Lancement Double d'Ariane 5. The SYLDA is located inside the launcher fairing (see fig.5) and allows for multiple payloads to be simultaneously launched. A 1/4 scale of the real system is to be computed. The dimensions of the modelled acoustic cavity surrounded by the structure are shown in fig.6.

The SYLDA comprises three main substructures, two conical and a cylindrical one all made of an orthotropic sandwich material. The sandwich structure has $1 \mathrm{~mm}$ thick facesheets made of Material II (see Table 1) and a 12.7mm thick core made of Material III. In Table 1 coordinate $a$ coincides with the axial direction of the shell, $c$ with the circumferential direction and $r$ with the radial one. The structure is modelled by coupling Mindlin-Reissner type shell FE for the facesheets to 3D solid elements for modelling the core. The FE model comprises 22710 structural DoF and 35465 pressure DoF in total (see fig.7).

A $\delta$-correlated (ROR) excitation is initially applied on the dry side of the cylindrical subsystem, using loads of equal magnitude and of random phase and position. A TBL excitation towards the axial direction of the structure is then modelled using the equivalent coherence function presented in sec.3. Firstly, the pressure level for a central cavity node is calculated in order to validate the ability of the SOAR to accurately predict the response of a single DoF. The 


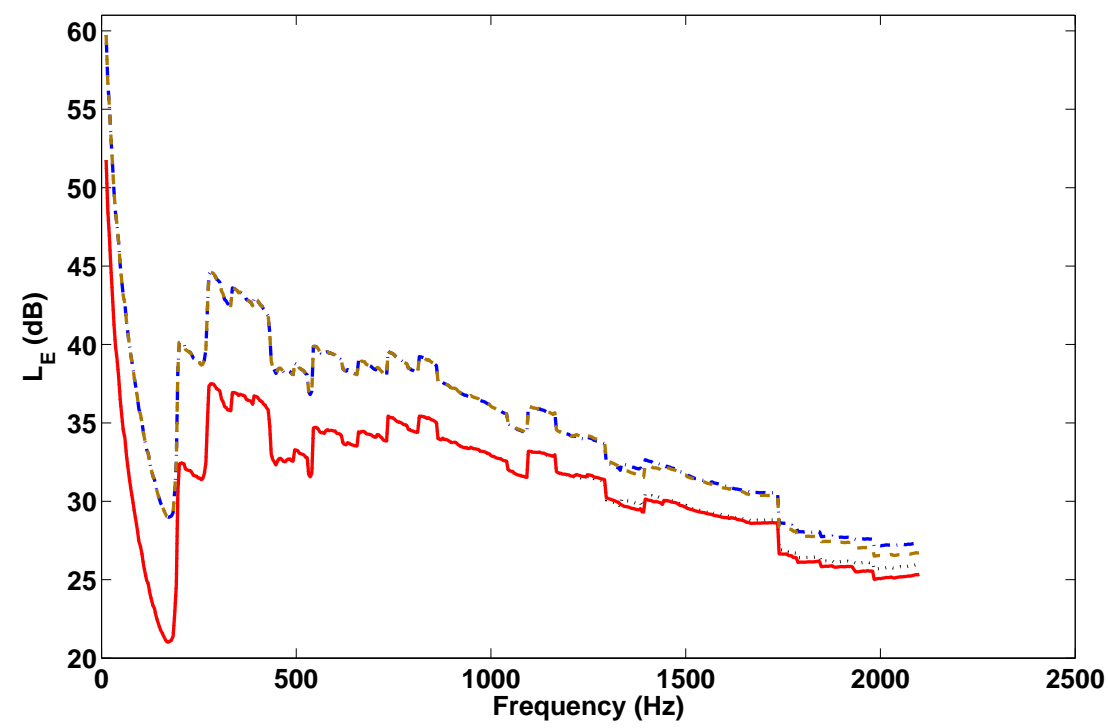

Figure 4: Energy of the cavity subsystem under a TBL excitation of 0.9 Mach applied on the panel: Layer propagating towards $x$ : FE direct solution $(\cdots)$, SOAR method $(-)$, Layer propagating towards $y$ : FE direct solution $(\cdot--\cdot)$, SOAR method (--)

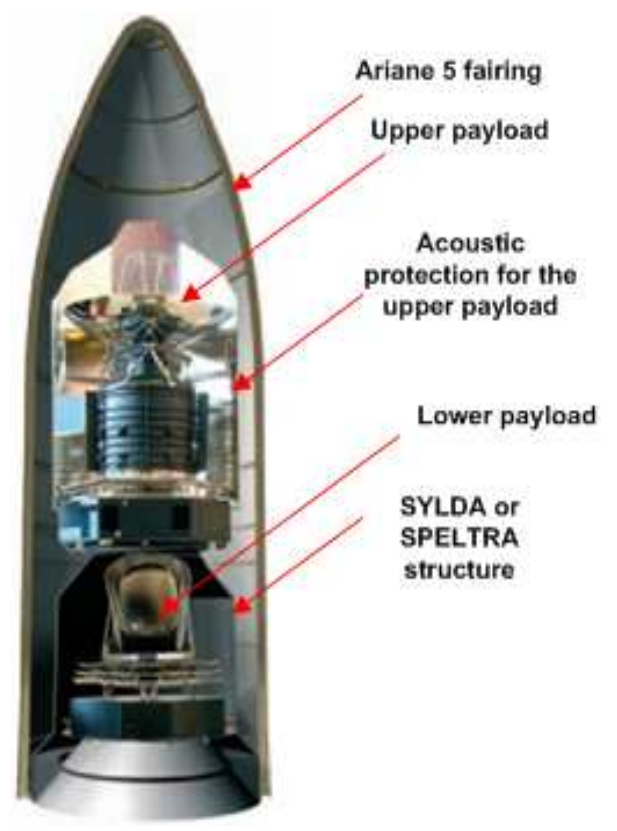

Figure 5: An illustration of the Ariane 5 spacecraft 


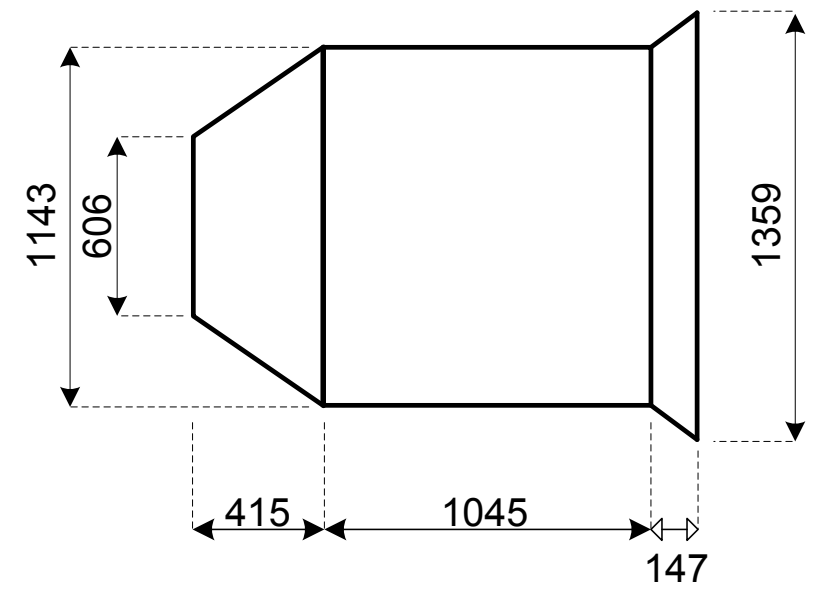

Figure 6: Dimensions of the modelled SYLDA configuration

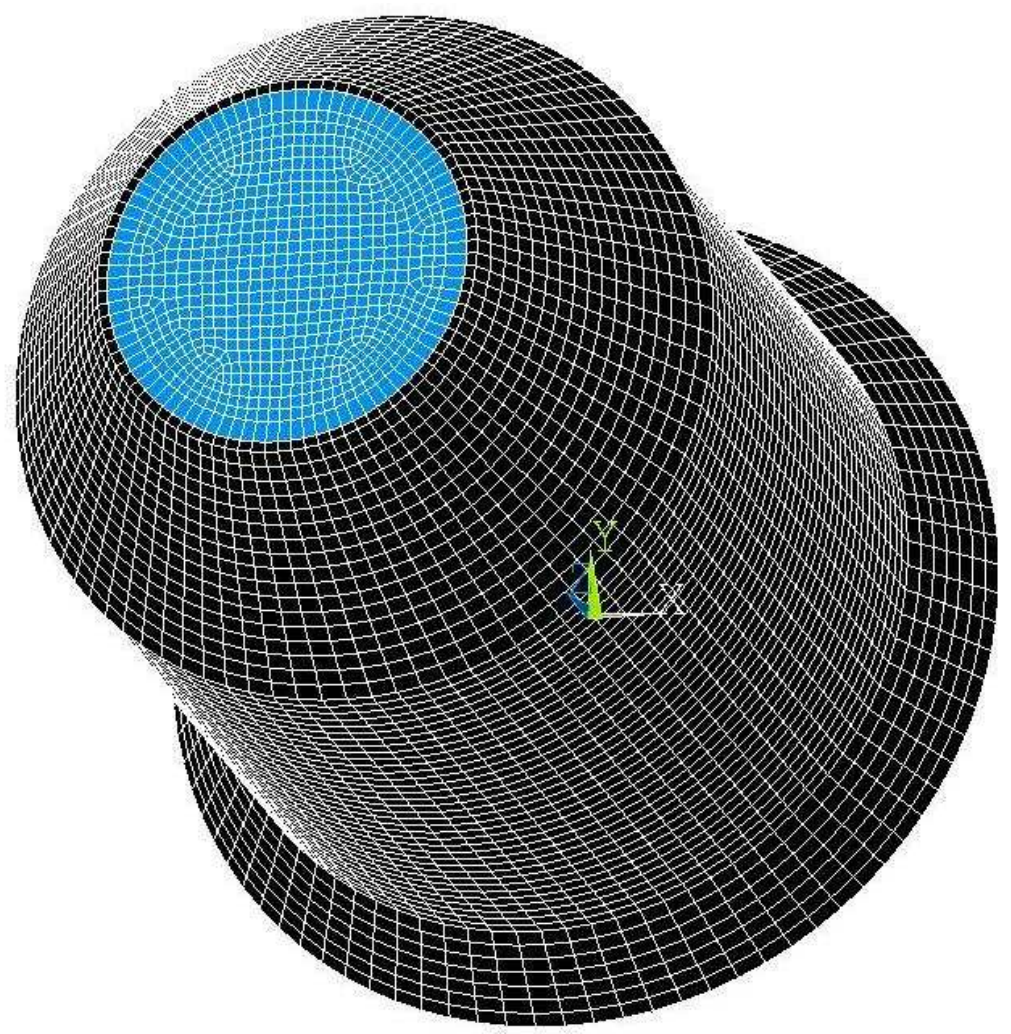

Figure 7: Caption of the FE SYLDA (black) and cavity (blue) subsystems 


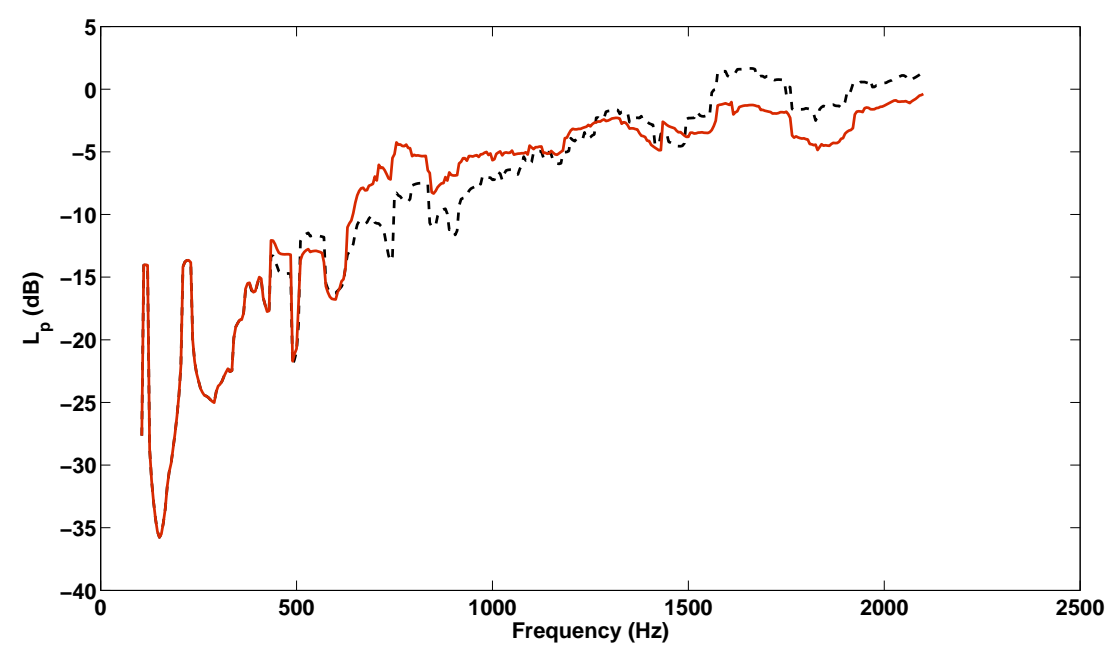

Figure 8: Acoustic pressure level at $0,0,0.7 \mathrm{~m}$ of the payload cavity when a ROR excitation is applied on the cylindrical subsystem: FE direct solution (- $)$, SOAR reduction (-)

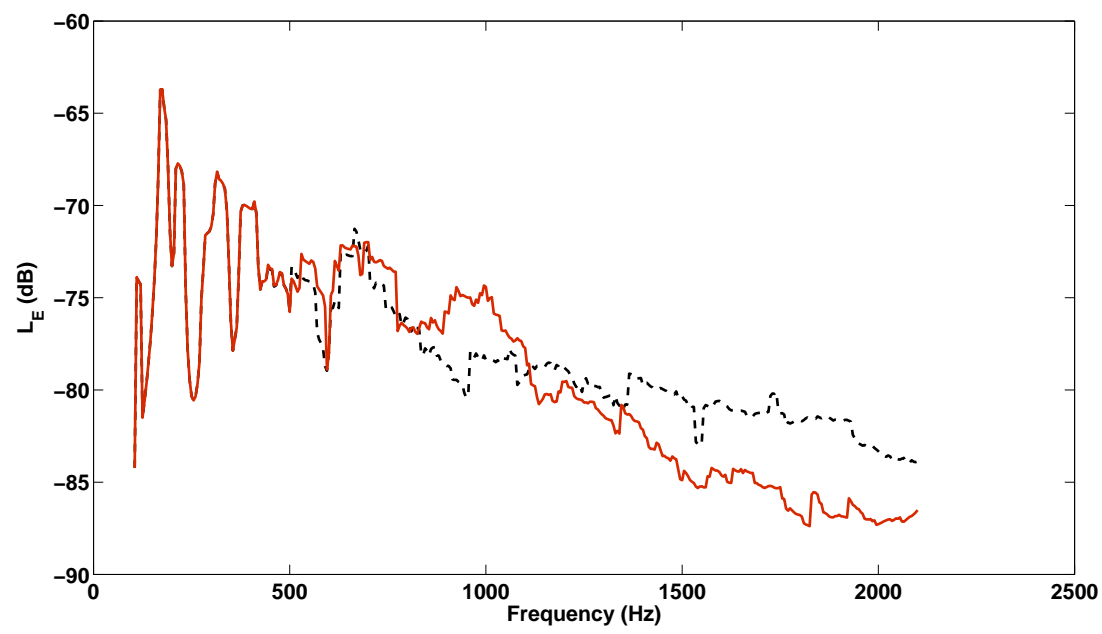

Figure 9: Acoustic energy level inside the payload cavity when a TBL excitation is applied on the cylindrical subsystem: FE direct solution (- -), SOAR reduction (-)

DoF coordinates are $(0 \mathrm{~m}, 0 \mathrm{~m}, 0.7 \mathrm{~m})$. The reference pressure is pref $=20 \mu \mathrm{Pa}$. The calculated SPL is shown in fig.8. The size of the reduced model is $m=500$ and the expansion point of the computation is $s_{0}=2100 \mathrm{~Hz}$ for all the results shown below. The results are averaged over one-twelfth octave frequency bands. Excellent agreement is observed in the low and medium frequency ranges between the FE solution and the SOAR prediction. The largest divergence of the approximation occurs at higher frequencies $(f=1650)$ and is equal to $3.5 \mathrm{~dB}$.

The acoustic energy level within the cavity when a TBL excitation is applied on the cylindrical subsystem is eventually computed. The result is presented in fig.9. As expected, the results are in a very good agreement up until $500 \mathrm{~Hz}$. The largest divergence is observed at $1900 \mathrm{~Hz}$, and is equal to $5.3 \mathrm{~dB}$. Higher accuracy can be obtained in the high frequency range by increasing $m$.

\section{Discussion on the resulting computational efficiency of the approach}

The ability of the SOAR reduction method to accurately model a structural acoustic system was exhibited in the above presented results. The calculation time for each of the above ex- 
Table 2: Calculation times

\begin{tabular}{|l|l|l|}
\hline \hline & $\begin{array}{l}\text { Orthotropic panel } \\
(m=2000)\end{array}$ & $\begin{array}{l}\text { SYLDA } \\
(m=500)\end{array}$ \\
\hline $\begin{array}{l}\text { Full FE solution } \\
\begin{array}{l}\text { Calculation of the Krylov subspace + so- } \\
\text { lution }\end{array}\end{array}$ & $\begin{array}{l}204 \mathrm{~min} \\
28 \mathrm{~min}\end{array}$ & $\begin{array}{l}934 \mathrm{~min} \\
54 \mathrm{~min}\end{array}$ \\
$\begin{array}{l}\text { Calculation of the Krylov subspace + so- } \\
\text { lution }+10 \% \text { sampling over acoustic DoF }\end{array}$ & $23 \mathrm{~min}$ & - \\
\hline
\end{tabular}

hibited calculations is presented in Table 2. It can therefore be concluded that the approach can drastically reduce the computation time while not having an important impact on the accuracy of the prediction. The total number of computed frequencies is 523. All calculations were done using a server of two, quadruple core Xeon E5343 processors, with 8Gb of RAM memory available. The direct FEM solution was done using a Lanczos algorithm within the software ANSYS 12.1, while the calculation of the Krylov subspace and the reduced solution was done using MATLAB 7.9.0. A total reduction of $88.7 \%$ was observed for the orthotropic panel system, while the reduction was equal to $94.2 \%$ in the case of the SYLDA structure. This time reduction is expected to be greater for larger industrial systems.

\section{Conclusions}

Concluding on the presented work, a structural acoustic system modelled with FE was reduced using the SOAR approach, and its broadband response to distributed aeroacoustic loads was calculated. The energy levels of the subsystems were calculated within an SEA-like approach. Summarizing the most important concluding points: 1) The approach can be used independently of the geometry and the structural acoustic coupling nature of the system as there is no need for analytical expressions of the dynamic characteristics of the subsystems. The structure may be composite and complex. The advantages of a FE modelling are therefore preserved. 2) Excellent agreement is observed in the low-frequency range for the SOAR estimation and the direct FE solution. The error of the approximation generally increases with respect to frequency. More vectors should therefore be added in the Krylov subspace in order to retain the error in a given level. 3) A cut-off frequency range was observed, during which the error of the reduced system approximation raises by several orders of magnitude. Increasing the dimension of the reduced model can delay the appearance of this cut-off frequency. 4) The calculation times are drastically reduced when the reduction is applied. Moreover, further reduction can be achieved by sampling over the acoustic DoF that are calculated for the application of the SEA-like approach.

\section{Acknowledgements}

The authors would like to gratefully acknowledge the ITN Marie Curie project GA-214909 'MID-FREQUENCY: CAE Methodologies for Mid-Frequency Analysis in Vibration and Acoustics' for the financial support.

\section{REFERENCES}

[1] W. Desmet, "Mid-frequency vibro-acoustic modelling: Challenges and potential solutions," in Proceedings of the 2002 International Conference on Noise and Vibration Engi- 
neering, ISMA, pp. 835-862, 2002.

[2] B. Mace, "Statistical energy analysis, energy distribution models and system modes," Journal of Sound and Vibration, vol. 264, no. 2, pp. 391-409, 2003.

[3] S. P. S. Maluski and B. M. Gibbs, "Application of a finite-element model to low-frequency sound insulation in dwellings," Journal of the Acoustical Society of America, vol. 108, no. 4, pp. 1741-1751, 2000.

[4] M. D. C. Magalhaes and N. S. Ferguson, "Acoustic-structural interaction analysis using the component mode synthesis method," Applied Acoustics, vol. 64, no. 11, pp. 1049-1067, 2003.

[5] J. Pan and D. A. Bies, "The effect of fluid-structural coupling on sound waves in an enclosure - Theoretical part," Journal of the Acoustical Society of America, vol. 87, no. 2, pp. 691-707, 1990.

[6] M. de Rochambeau, M. Ichchou, and B. Troclet, "Modeling of random aerodynamic loads applied on fluid-structure coupled systems using rain-on-the-roof equivalent excitation," Engineering Computations (Swansea, Wales), vol. 28, no. 4, pp. 472-491, 2011.

[7] B. R. Mace, "Statistical energy analysis: Coupling loss factors, indirect coupling and system modes," Journal of Sound and Vibration, vol. 279, no. 1-2, pp. 141-170, 2005.

[8] C. Fredö, "A SEA-like approach for the derivation of energy flow coefficients with a finite element model," Journal of Sound and Vibration, vol. 199, no. 4, pp. 645-666, 1997.

[9] B. Troclet, B. Hiverniau, M. N. Ichchou, L. Jezequel, K. Kayvantash, and T. Bekkour, "FEM / SEA hybrid method for predicting mid and high frequency structure-borne transmission," The Open Acoustics Journal, vol. 2, pp. 45-60, 2009.

[10] L. Peretti and E. Dowell, "Asymptotic modal analysis of a rectangular acoustic cavity excited by wall vibration," AIAA Journal, vol. 30, no. 5, pp. 1191-1198, 1992.

[11] M. D. C. Magalhaes and N. S. Ferguson, "The development of a component mode synthesis (cms) model for three-dimensional fluid-structure interaction," Journal of the Acoustical Society of America, vol. 118, no. 6, pp. 3679-3690, 2005.

[12] A. C. Antoulas, "Approximation of large-scale dynamical systems," 2005.

[13] K. Willcox, J. Peraire, and J. White, "An Arnoldi approach for generation of reduced-order models for turbomachinery," Computers and Fluids, vol. 31, no. 3, pp. 369-389, 2001.

[14] J. S. Han, E. B. Rudnyi, and J. G. Korvink, "Efficient optimization of transient dynamic problems in MEMS devices using model order reduction," Journal of Micromechanics and Microengineering, vol. 15, no. 4, pp. 822-832, 2005.

[15] R. Srinivasan Puri, D. Morrey, A. J. Bell, J. F. Durodola, E. B. Rudnyi, and J. G. Korvink, "Reduced order fully coupled structural-acoustic analysis via implicit moment matching," Applied Mathematical Modelling, vol. 33, no. 11, pp. 4097-4119, 2009. 
[16] Z. Bai and Y. Su, "SOAR: A second-order Arnoldi method for the solution of the quadratic eigenvalue problem," SIAM Journal on Matrix Analysis and Applications, vol. 26, no. 3, pp. 640-659, 2005.

[17] G. M. Corcos, "Resolution of pressure in turbulence," Journal of the Acoustical Society of America, vol. 35, no. 2, pp. 192-199, 1963.

[18] D. M. Chase, "Modeling the wavevector-frequency spectrum of turbulent boundary layer wall pressure," Journal of Sound and Vibration, vol. 70, no. 1, pp. 29-67, 1980.

[19] B. M. Efimtsov, "Characteristics of the field of turbulent wall pressure fluctuations at large Reynolds numbers," Soviet Physics Acoustics, vol. 28, no. 4, pp. 289-292, 1982.

[20] G. Maidanik, "Use of delta function for the correlations of pressure fields," Journal of the Acoustical Society of America, vol. 33, no. 11, pp. 1598-1609, 1961.

[21] F. Birgersson and S. Finnveden, "A spectral super element for modelling of plate vibration. part 2: Turbulence excitation," Journal of Sound and Vibration, vol. 287, no. 1-2, pp. 315$328,2005$.

[22] M. Ichchou, B. Hiverniau, and B. Troclet, "Equivalent 'rain on the roof' loads for random spatially correlated excitations in the mid-high frequency range," Journal of Sound and Vibration, vol. 322, no. 4-5, pp. 926 - 940, 2009.

[23] J. Guyader, C. Boisson, and C. Lesueur, "Energy transmission in finite coupled plates, part I: Theory," Journal of Sound and Vibration, vol. 81, no. 1, pp. 81-92, 1982.

[24] G. C. Everstine, "Finite element formulatons of structural acoustics problems," Computers and Structures, vol. 65, no. 3, pp. 307-321, 1997.

[25] F. G. Leppington, E. G. Broadbent, and K. H. Heron, "Acoustic radiation efficiency of rectangular panels," in Proceedings of The Royal Society of London, Series A: Mathematical and Physical Sciences, vol. 382, pp. 245-271, 1982.

[26] A. Craggs, "The transient response of a coupled plate-acoustic system using plate and acoustic finite elements," Journal of Sound and Vibration, vol. 15, no. 4, pp. 509-528, 1971.

[27] C. de Villemagne and R. E. Skelton, "Model reductions using a projection formulation.," International Journal of Control, vol. 46, no. 6, pp. 2141-2169, 1987.

[28] T.-J. Su and R. R. Craig Jr., "Model reduction and control of flexible structures using Krylov vectors," Journal of Guidance, Control, and Dynamics, vol. 14, no. 2, pp. 260267, 1991.

[29] J. Lampe and H. Voss, "Second-order Arnoldi: Reduction application to some engineering problems," Proceedings of the XVI-th Summer School on Software and Algorithms of Numerical Mathematics, pp. 153-173, 2005.

[30] B. Salimbahrami and B. Lohmann, "Order reduction of large scale second-order systems using Krylov subspace methods," Linear Algebra and Its Applications, vol. 415, no. 2-3, pp. 385-405, 2006. 
[31] Y. Yue and K. Meerbergen, "Using model order reduction for design optimization of structures and vibrations," TW Reports TW566, Department of Computer Science, 2010. 